\title{
Bioabsorbable Barrier Membrane Combined with rhBMP-2 Improved Bone Formation in an Experimental Model of Compromised Healing But Was Not Superior to rhBMP-2 Alone
}

\author{
Henrik Eckardt ${ }^{1, *}$, Knud Stenild Christensen ${ }^{2}$, Martin Lind ${ }^{3}$, Ebbe Stender Hansen ${ }^{3}$, Ivan Hvid ${ }^{3}$ \\ ${ }^{1}$ Trauma Division, Orthopedic Department, Copenhagen University Hospital, Rigshospitalet, Denmark; ${ }^{2}$ Department of Orthopedic \\ Surgery, Aalborg Hospital, Aalborg, Denmark; ${ }^{3}$ Department of Orthopedic Surgery, Aarhus University Hospital, Aarhus, Denmark. \\ Email: henrikeckardt@gmail.com
}

Received December $9^{\text {th }}, 2013$; revised January $12^{\text {th }}, 2014$; accepted January $20^{\text {th }}, 2014$

Copyright (C 2014 Henrik Eckardt et al. This is an open access article distributed under the Creative Commons Attribution License, which permits unrestricted use, distribution, and reproduction in any medium, provided the original work is properly cited. In accordance of the Creative Commons Attribution License all Copyrights (C) 2014 are reserved for SCIRP and the owner of the intellectual property Henrik Eckardt et al. All Copyright (C) 2014 are guarded by law and by SCIRP as a guardian.

\section{ABSTRACT}

Objective: Bioabsorbable barrier membranes placed over alveolar ridge bone defects are routinely used in dental surgery to promote bone formation. Combining these osteoconductive membranes with osteoinductive Bone Morphogenetic Proteins could prove useful in long bone fracture treatment. The hypothesis was tested in a clinically relevant model of compromised healing. Methods: Four groups of 8 rabbits underwent unilateral mid-tibial osteotomy, excision of periosteum and endosteum, and plate fixation. One group had rhBMP-2 deposited between the bone ends and Membrane wrapped around the osteotomy, the second group had Membrane wrapped around the osteotomy, the third group had rhBMP-2 placed between the bone ends, and the fourth group received no additional treatment. Results: After 7 weeks, callus size and blood flow were significantly higher in the Membrane+rhBMP-2 group than in the rhBMP-2 treated group, but torsion to failure test showed no significant difference. Membrane treatment and no treatment led to non-union. Conclusion: Absorbable barrier membrane combined with rhBMP-2 enhances bone formation, but has no advantage to rhBMP-2 alone. Membrane alone wrapped around the osteotomy was unable to prevent non-union formation.

\section{KEYWORDS}

Bioabsorbable Barrier Membrane; Bone Morphogenetic Protein 2; Non Union; Pseudartrhrosis; Rabbit; Bone Blood Flow; Torsion to Failure

\section{Introduction}

Recombinant human Bone Morphogenetic Proteins have proven efficacy in experimental as well as clinical settings for both fracture healing enhancement and treatment of tibial nonunion, and are the best available alternative to bone grafts for enhancement of fracture healing $[1,2]$. However, the clinical effect is dose dependent, and the high dose required inducing adequate bone formation raises concern regarding safety and cost [3].

Barrier membranes have been used in dentistry for

"Corresponding author. years. They assist in the healing of periodontal defects by providing a favourable environment for bone regeneration, a scaffold for migrating osteoprogenitor cells, and by excluding soft tissue ingrowth, and they are reabsorbed within 3 months [4,5]. In comminuted fractures with bone loss and in other fractures with poor healing potential due to severed periosteum, membranes could substitute the osteoconductive effect of the periosteum and aid in containment of the recombinant growth factor, that is deposited in the interfragmentary space.

The aim of this study was to test whether the combined action of an absorbable barrier membrane and 
rhBMP-2 was superior to rhBMP-2 alone with respect to enhancement of bone healing.

The bone healing model that we used was a validated experimental model of compromised healing in rabbits. After a mid-tibial osteotomy, we removed periosteum and endosteum proximal and distal to the osteotomy thus compromising the bone healing process so that fibrocartilagineous tissue formed between the bone ends instead of callus, and a non-union developed that, histologically, is similar to an atrophic non-union in human [6,7]. We therefore consider the model clinical relevant.

We hypothesized that rhBMP-2 combined with an absorbable barrier membrane would provide the osteotomised bone ends of our model with a stronger osteoinductive and osteoconductive stimulus than rhBMP-2 alone thus resulting in faster and stronger bone consolidation. We added two control groups to explore the effect of barrier membrane treatment: one control group received only barrier membrane treatment and one control group received no treatment. Only the relevant comparisons between the four groups were conducted, and the design of the study is thus an incomplete factorial design.

The present experiment was one arm of a larger experiment and the results from the rhBMP-2 group and from the group that received hyaluronan carrier alone have been published previously [8]. The previous study focused on the effect of rhBMP-2 in the non-union model and compared rhBMP-2 with no treatment.

\section{Methods}

\subsection{Design}

Skeletally mature New Zealand White rabbits, 5 - 6 months old, allocated to 4 groups of 8, underwent a standard non-union operation. Group I received interfragmentary rhBMP-2 delivered in a hyaluronan gel carrier and had Membrane (Gore Resolut XT6 ${ }^{\circledR}$ ) wrapped around the osteotomy. Group II received hyaluronan gel in the osteotomy gap and Membrane implantation, and group III received interfragmentary rhBMP-2 delivered in a hyaluronan gel. Group IV had only hyaluronan gel deposited between the bone ends. The experiment was approved by the Danish Committee on Animal Experimentation (approval \# 2002/561-501).

Group I was denoted "Membrane+rhBMP-2", group II was denoted "Membrane", group III was denoted "rhBMP-2" and group IV was denoted "Vehicle".

\subsection{Membrane}

The Membrane was a commercially available product used in dentistry (Figure 1). The Gore Resolut XT6 ${ }^{\circledR}$,

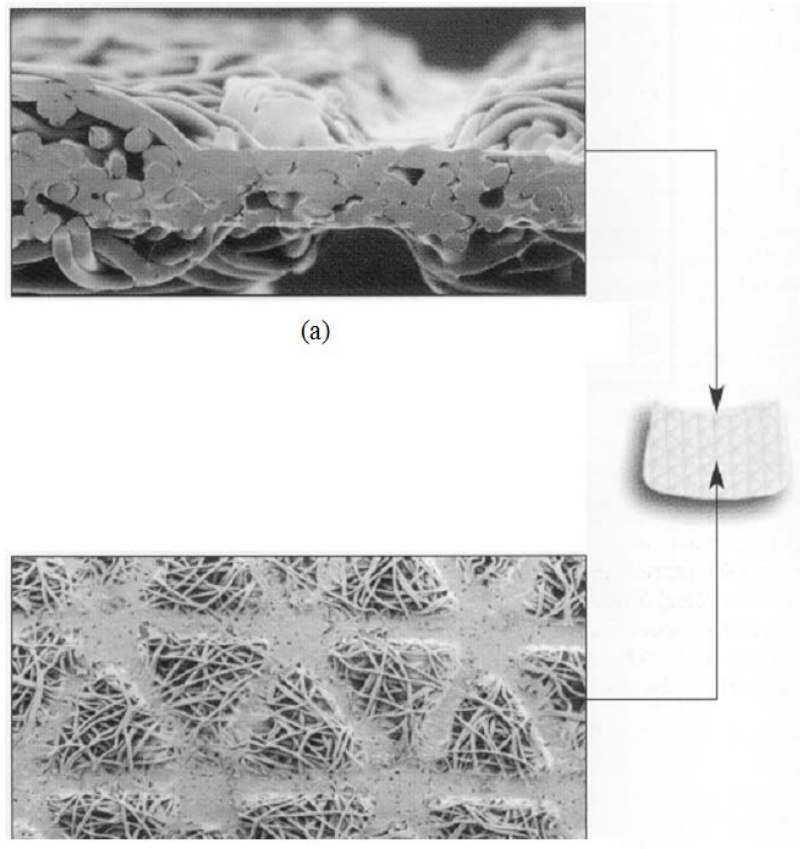

(b)

Figure 1. (a) Cross sectional view of membrane: The membrane is a three-layer structure, including two random fiber matrices on both the gingival and defect sides of a cell occlusive film. (b) Surface view of membrane: TRIMETRIC pattern increases membrane flexibility.

was a $25 \mathrm{~mm} \times 20 \mathrm{~mm} \mathrm{100 \%}$ synthetic membrane composed of bioabsorbable glycolide, trimethylene carbonate copolymer fiber and lactide copolymer. The three-layer structure includes random fiber matrices on both gingival and defect sides of a cell occlusive film, thus separating the different tissue types on the gingival and defect side. Membranes maintain their mechanical integrity for 8-10 weeks in humans, degrade into carbon dioxide and water and initiate mild inflammation on degradation. The membrane was fastened with Gore Resolut ${ }^{\circledR}$ suture; an absorbable synthetic polyglycolic acid suture (Gore \& Associates, Flagstaff, AZ, USA).

\subsection{Growth Factor and Vehicle}

Recombinant human Bone Morphogenetic Growth Factor was donated by Yamanouchi Europe BV, Leiderdorp, The Netherlands as a frozen $1 \mathrm{mg} / \mathrm{ml}$ solution in $4.5 \mathrm{mM}$ sodium glutamate buffer $\mathrm{pH}$ 4.5. The material was thawed slowly to refrigeration temperature and an injectate of $0.5 \mathrm{ml}$ containing $200 \mu \mathrm{g}$ rhBMP-2 was prepared by adding $0.2 \mathrm{ml}$ stock solution and $0.1 \mathrm{ml}$ saline to 0.2 $\mathrm{ml}$ hyaluronan gel, $1.4 \%$ sodium hyaluronate (I-Visc Plus $^{\circledR}$, I-med pharma). This was then mixed in a syringe for several minutes before deposition. Hyaluronic acid is the natural lubricant of joint fluid, it is a $100 \%$ synthetic and viscous gel that is injectable, it has been used in 
ophthalmic surgery for years and has an osteoinductive action itself [9].

\subsection{Surgical Procedure}

A validated nonunion model, modified through the use of plates instead of external fixation, was used and found to be robust and reproducible in pilot studies [6]. Using an anteromedial approach in anaesthetized rabbits, we osteotomised the right distal tibia diaphysis. We then striped the periosteum and endosteum $15 \mathrm{~mm}$ proximal and distal to the osteotomy, and fixed a 5-hole 3/8 Orthofix plate on the anterolateral aspect of the tibia, leaving a 2 $\mathrm{mm}$ gap between the osteotomised bone ends. After saline irrigation, interfragmentary rhBMP-2 or vehicle alone was deposited with a syringe and Membrane was tightly wrapped around the tibia and fastened with 2 sutures across the plate according to the group involved. The wound was sutured in 2 layers, and the rabbits allowed free ambulation.

\subsection{Procedures after Sacrifice, in Brief}

After 7 weeks we anaesthetised the rabbits and measured blood flow by the labelled microsphere injection technique. The rabbits were sacrificed and the bone of both legs dissected free for soft tissue, the plate was removed and the distal diaphyses of both hindlegs were isolated for counting in a gamma counter and then frozen. After thawing, a micro-CT-scan was performed and both ends of the sample were embedded in bone cement for torsional testing.

\subsection{Blood Flow Measurement}

Blood flow measurements were performed according to the technique of Hales [10]. In brief $4 \times 10^{6}$ Strontium-85 labelled $15 \mu \mathrm{m}$ microspheres were injected into the left ventricle of the heart via the left common carotid artery, and reference samples withdrawn through the right common carotid artery via a pump. The microspheres were injected over 30 seconds, reference blood samples taken over 2 minutes and the animals sacrificed by an overdose of barbiturate. Mean blood pressure and heart rate were monitored to document cardiovascular stability. The bones were cleaned from soft tissue and the distal diaphysis of the right tibia, comprising the osteotomy site, was isolated and counted in a gamma counter with the reference blood sample (Packard Cobra 5000; GMI, inc.; Clearwater; MA, USA). Counts in each channel were corrected for background radiation.

An extensive review of procedures and assumptions for the microsphere method has been given elsewhere [11]. Blood flow in a region is calculated from SR $\mathrm{C}_{\mathrm{REG}} / \mathrm{C}_{\mathrm{REF}}$, where $\mathrm{SR}$ and $\mathrm{C}_{\mathrm{REF}}$ denotes sampling rate and activity of the reference sample, respectively, and $\mathrm{C}_{\mathrm{REG}}$ denotes activity of the region. Regional blood flow (RBF) is standardized to $100 \mathrm{mg}$ of tissue.

\subsection{Torsional Testing}

The free ends of the distal diaphysis that contained the osteotomy site was embedded in bone cement and fastened in the test device. The mid $20 \mathrm{~mm}$ were left free and tested in torsion using a 858 Bionix hydraulic material-testing machine, computer-controlled by the software TestStar II Operation System (MTS Systems Cooperation, Minneapolis, Minnesota, USA). Torsional failure load, failure angle and energy absorbed before fracture were recorded from the load-displacement curve.

\subsection{Micro CT}

The specimens were scanned with a high-resolution microtomographic system ( $\mu$ CT 20, Scanco Medical AG., Zurich, Switzerland) in a multislice mode rendering a resolution of $20 \mu \mathrm{m}$. Bone was isolated with the help of an automated threshold procedure [12]. From a horizontal slice midway between the osteotomised bone ends the cross sectional area of callus was measured (tCSA).

\subsection{Serum Cytology}

In order to quantify a systemic inflammatory reaction following degradation of the implanted membranes, blood collected prior to sacrifice was analysed for haemoglobin, sedimentation reaction, blood platelet count, white cell count and differential count using automated routine analyses calibrated for use in man.

\subsection{Anaesthesia}

Balanced anaesthesia was obtained by the mixture of 20 $\mathrm{ml}$ Ketamine (50 mg/ml, Pfizer), $2.5 \mathrm{ml}$ Lidocaine (20 $\mathrm{mg} / \mathrm{ml}$, Xylacaine ${ }^{\circledR}$, Astra) and $1 \mathrm{ml}$ Acepromazine (10 $\mathrm{mg} / \mathrm{ml}$, Plegecid ${ }^{\circledR}$, Pharmacia \& Upjohn) administered as $23.5 \mathrm{ml} / \mathrm{kg}$ subcutaneous injections repeated every $1 / 2$ hour during the operation. Brupenorfen $(0.1 \mathrm{mg} / \mathrm{kg}$, Temgesic $^{\circledR}$, Reckitt \& Coleman) was used for postoperative pain relief for 2 - 3 days. Intracardial Pentobarbital injection (Mebumal ${ }^{\circledR}, 200 \mathrm{mg} / \mathrm{ml}$, Sygehus Apotekerne, Denmark) on anaesthetised animals secured pain free sacrifice.

\subsection{Data Analysis}

One way ANOVA followed by non-parametric MannWhitney $U$ test for 2 unpaired samples for comparison between groups was used due to the small sample size. When ANOVA yielded $\mathrm{p}>0.05$, no further tests were conducted and the result denoted not significant (n.s.) 
Retrospective power analysis could not be performed due to the limited number in each group.

\section{Results}

\subsection{General}

Membrane implantation and rhBMP-2 deposition were uneventful. All rabbits tolerated the experimental procedure, none had infections and they ambulated freely a few days after surgery. There was no significant difference in median body mass or weight gain between groups. Due to technical difficulties 2 bone specimens were lost for torsional testing and micro-CT, and in another 8 rabbits the bone blood flow measurements were unsuccessful, thus leaving 24 rabbits for bone blood flow analyses.

\subsection{Macroscopic Evaluation}

Macroscopic evaluation of the Membrane + rhBMP-2 and the rhBMP-2 group revealed an osteotomy gap completely filled with bone, the distal diaphysis and the plate embedded in a porous, spongy and blood-rich callus. Manual assessment of stiffness revealed solid union in both groups. In the Membrane group and in the Vehicle group, only small amounts of interfragmentary bone had formed, and 5 and 4 rabbits respectively proceeded to a fibrous, mobile union. The membrane was not macroscopically visible in any groups.

\subsection{Radiographic Examination}

Radiographic examination showed interfragmentary radio-opacity in the Membrane + rhBMP-2 group and the rhBMP-2-group, the continuity between bone ends almost fully restored, whereas the Membrane group and Vehicle group displayed a radiolucent gaps without restoration of the continuity (Figure 2).

\subsection{Torsional Testing}

Table 1 and show that median failure moment, angular displacement and energy absorbed at failure in the Membrane+rhBMP-2 was significantly higher than in the Membrane group. Energy at failure was 57\% higher in the rhBMP-2-group than in the Membrane + rhBMP-2 group, but the difference was not significant $(\mathrm{p}=0.06)$. There was no difference between biomechanical properties of the bone formed in the Membrane and the Vehicle group. In 2 rabbits technical difficulties resulted in missing torsional recording.

\subsection{Blood Flow Measurement}

Table 2 shows that regional blood flow (RBF) was high-
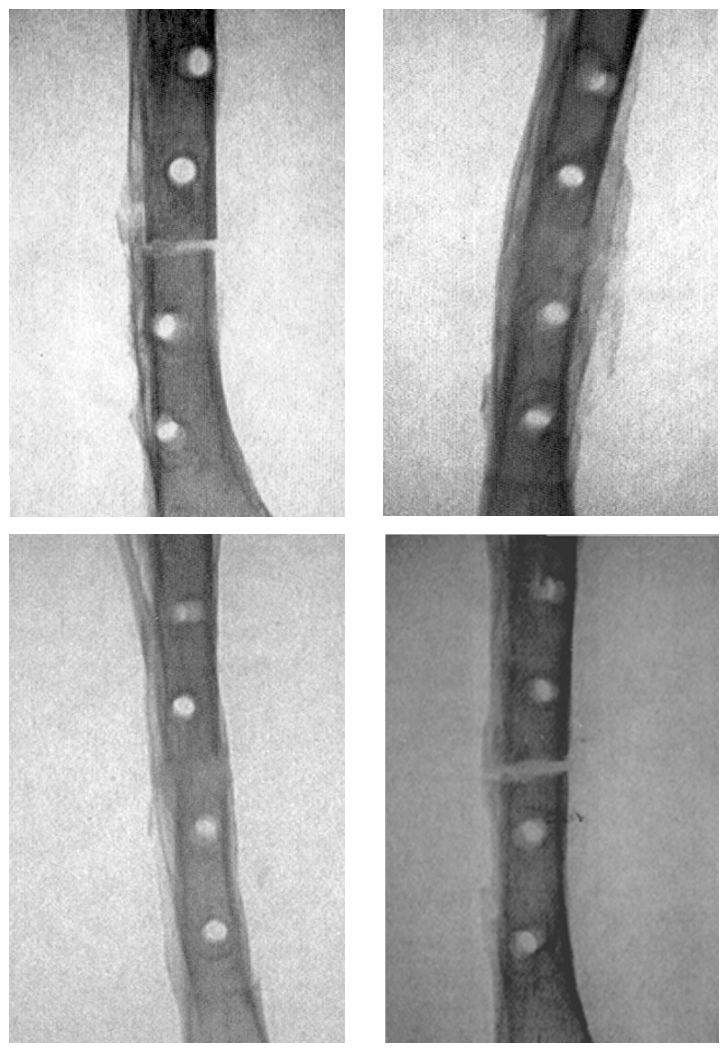

Figure 2. Antero-posterior $x$-ray of rabbit tibia osteotomies 7 week after a standard nonunion operation. Upper row to the left a Membrane+rhBMP-2 treated osteotomy, upper row to the right a Membrane treated, lower row to the left a rhBMP-2 treated osteotomy, and lower row the right a Vehicle treated osteotomy.

er in the Membrane + rhBMP-2 treated group than in the rhBMP-2 treated group. There was no difference between Membrane + rhBMP-2 and Membrane treated osteotomies and no difference between Membrane and Vehicle treated osteotomies. One way ANOVA test for mean blood pressure and heart rate revealed $\mathrm{p}>0.05$. Thus, we found no significant difference between the basic hemodynamic parameters in the 4 groups. In 8 rabbits, blood flow measurements were not successful due to technical difficulties.

\subsection{MicroCT}

Table 1 shows that the total callus cross sectional area (tCSA), which is the total callus covered area including bone marrow, was significantly higher in the Membrane + rhBMP-2 group than in the rhBMP-2-group. The Membrane group and the Vehicle group displayed significantly smaller tCSA than the Membrane + rhBMP-2 and rhBMP-2 groups and there was no difference between tCSA in Membrane and Vehicle group. A representative interfragmentary 3D-reconstruction of an interfragmentary slice from each group is shown in Figure 3. 
Table 1. Torsion to failure test and static histomorphometry. Median followed by $25 \%$ and $75 \%$ quartiles in brackets. One way ANOVA followed by Mann-Whitney $U$ test was used to calculate p-value. $n$ designates the number of animals available for analysis. tCSA denoted total cross sectional area. Reduced number of rabbits due to technical difficulties during biomechanical tests.

\begin{tabular}{|c|c|c|c|c|c|c|c|}
\hline & \multirow{2}{*}{$\begin{array}{c}\text { Group I }(\mathrm{n}=7) \\
\text { Membrane + rhBMP-2 }\end{array}$} & \multirow{2}{*}{$\begin{array}{l}\text { Group II }(\mathrm{n}=8) \\
\text { Membrane }\end{array}$} & \multirow{2}{*}{$\begin{array}{l}\text { Group III }(\mathrm{n}=7) \\
\text { rhBMP-2 }\end{array}$} & \multirow{2}{*}{$\begin{array}{l}\text { Group IV }(\mathrm{n}=8) \\
\text { Vehicle }\end{array}$} & \multicolumn{3}{|c|}{$\mathrm{p}$ value } \\
\hline & & & & & Group I vs. II & Group I vs. & III Group II vs. IV \\
\hline Failure load/Nmm & $700(700$ - 975) & $0(0-612)$ & $1000(925$ - 1200) & $400(0-121)$ & 0.007 & 0.1 & 0.5 \\
\hline $\begin{array}{c}\text { Energy absorbed at } \\
\text { failure/degree x Nmm }\end{array}$ & $8000(4200-11700)$ & $2800(0-5800)$ & $12600(8500-16300)$ & $1800(0$ - 5700) & 0.02 & 0.06 & 0.9 \\
\hline Failure angle/degree & $9.5(7.2-21)$ & $0(0-13)$ & $11(6.2-17)$ & $2(0-6.7)$ & 0.06 & 0.8 & 0.9 \\
\hline $\mathrm{tCSA} / \mathrm{mm}^{2}$ & $80(67-87)$ & $47(32-55)$ & $61(50-65)$ & $39(8-53)$ & 0.004 & 0.01 & 0.7 \\
\hline
\end{tabular}

Table 2. Regional Blood Flow (RBF) and hemodynamic parameters during microsphere injection. Median followed by $25 \%$ and $75 \%$ quartiles in brackets. One way ANOVA followed by Mann-Whitney $U$ test was used to calculate p-value. n designated the number of animals. ANOVA yielded non-significant differences between groups for mean blood pressure and heart rate and no further tests were conducted. Reduced number of rabbits due to technical difficulties during blood flow measurements.

\begin{tabular}{|c|c|c|c|c|c|c|c|}
\hline & Group I $(\mathrm{n}=7)$ & Group II $(n=6)$ & Group III $(n=6)$ & Group IV $(n=5)$ & \multicolumn{3}{|c|}{$\mathrm{p}$ value } \\
\hline & $\begin{array}{c}\text { Membrane + } \\
\text { rhBMP-2 }\end{array}$ & Membrane & rhBMP-2 & Vehicle & Group I vs. II & Group I vs. & III Group II vs. IV \\
\hline $\mathrm{RBF}_{\text {distal diaphysis }} / \mathrm{ml} / \mathrm{min} / 100 \mathrm{~g}$ & $22(15-32)$ & $22(16-26)$ & $6(5-12)$ & $15(12-16)$ & 1 & 0.005 & 0.6 \\
\hline Mean blood pressure/mmHg & $64(61-73)$ & $65(60-76)$ & $53(45-63)$ & $71(62-80)$ & n.s. & n.s. & n.s. \\
\hline Heart rate/pm & $173(156-190)$ & $161(150-196)$ & $184(178-198)$ & $130(115-170)$ & n.s. & n.s. & n.s. \\
\hline
\end{tabular}
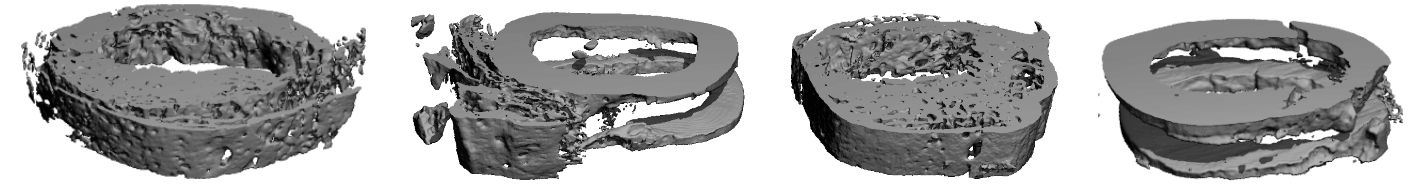

Figure 3. 3D reconstructed image of $3 \mathrm{~mm}$ high horizontal section of the osteotomy site, posterior view. To the left a Membrane + rhBMP-2 treated osteotomy, second from left a Membrane treated, third from left a rhBMP-2 treated, and to the right a Vehicle treated osteotomy 7 weeks after operation. Note the absence of interfragmentary callus in the Membrane group, and Vehicle group and the united and remodelled osteotomies in the Membrane + rhBMP-2 and rhBMP-2 group.

\subsection{Serum Cytology}

Haemoglobin, white blood cell count, sedimentation reaction and blood platelet count displayed in Table 3 showed no difference among groups.

\section{Discussion}

In the present experiment, barrier membranes alone failed to stimulate bone healing and prevent non-union formation. Synthetic absorbable membranes are used clinically for periodontal repair and for alveolar ridge augmentation. A multi-center randomized controlled clinical trial concluded that membranes sutured over alveolar defects increased alveolar bone stock by $40 \%$ when compared to defects that was covered by a standard flap alone [13]. The favourable results achieved by dentist have not been reproduced in relevant orthopaedic settings. In a comprehensive review of barrier membranes, Dimitriou et al. concludes that several questions still remain to be answered. The additive use of bone graft and the definition of the ideal membrane are unsolved issues and efficacy, safety, and cost effectiveness of barrier membranes must be established before clinical use can be recommended [14].

We found that the combination of rhBMP-2 and a barrier membrane prevented non-union formation and initiated the production of competent bone. Yet restoration of mechanical competence is the ultimate end point in bone healing experiments, and rhBMP-2 combined with a barrier membrane was not superior to rhBMP-2 in torsional testing. Implantation of the barrier membrane alone could not prevent non-union formation and biomechanical properties and blood flow in the group that had membrane wrapped around the osteotomy was not different from the group that received no treatment. Thus, our experiment failed to exhibit an osteopromotive effect of the membrane in a relevant orthopaedic experimental model. A review of the literature shows that $10 \mu \mathrm{g}$ rhBMP-2 deposited in a membrane covered rabbit 10 $\mathrm{mm}$ critical size radius defect stimulated bone formation in comparison to membrane alone, but the study did not include a control group that received growth factor alone 
Table 3. Serum cytology. Routine automated human analyses of rabbit blood collected prior to sacrifice.

\begin{tabular}{ccccc}
\hline & $\begin{array}{c}\text { Membrane + rhBMP-2 } \\
(\mathrm{n}=8)\end{array}$ & $\begin{array}{c}\text { Membrane } \\
(\mathrm{n}=8)\end{array}$ & $\begin{array}{c}\text { rhBMP-2 } \\
(\mathrm{n}=8)\end{array}$ & $\begin{array}{c}\text { Vehicle } \\
(\mathrm{n}=8)\end{array}$ \\
\hline Haemoglobin/mmol/l & $6.9(6.6-7.3)$ & $6.9(6.8-7.2)$ & $6.6(6.2-7.0)$ & $6.5(6.1-7.1)$ \\
Blood plates/10 $/ 1$ & $358(242-590)$ & $382(349-390)$ & $411(331-445)$ & $416(370-456)$ \\
Sedimentation & $1(0-1)$ & $1(0-1)$ & $1(0-1)$ & $1(0-1)$ \\
Leukocytes/10 $/ 1$ & $5.0(4.4-5.3)$ & $5,1(4.5-5.2)$ & $4.6(3.9-5.6)$ & $4.7(4.1-5.2)$ \\
Neutrocytes/\% & $0.25(0.2-0.5)$ & $0.3(0.1-0.8)$ & $0.55(0.23-0.92)$ & $0.25(0.1-0.4)$ \\
Lymphocytes/\% & $82(75-85)$ & $82(78-85)$ & $78(52-79)$ & $78(55-82)$ \\
Monocytes/\% & $11(7-19)$ & $14(9-16)$ & $17(15-37)$ & $14(12-18)$ \\
Eosinophilic/\% & $0.7(0.4-1.0)$ & $0.7(0.3-0.8)$ & $0.7(0.5-2.3)$ & $0.7(0.5-2.1)$ \\
Basophilic/\% & $3.9(3.1-4.4)$ & $3.5(2.5-4.9)$ & $3.1(2.5-3.9)$ & $3.9(3.3-4.2)$ \\
\hline
\end{tabular}

[15]. The deposition of $10 \mu \mathrm{g}$ rhBMP-2 in a mouse mandibular circular $5 \mathrm{~mm}$ defect covered by an absorbable membrane showed increased bone formation compared to vehicle deposition alone but was not different from rhBMP-2 deposition [16]. Bone formation following demineralised bone matrix powder deposition in a membrane covered $10 \mathrm{~mm}$ rabbit radius critical sized defect was superior to bone matrix powder alone and superior to membrane alone [17]. Our experiment supplements and supports the previous studies by using a relevant long bone model.

Both total callus area and blood flow of the callus were larger in the group that received membrane and rhBMP-2 treatment than in the group that received only rhBMP-2. The increased blood flow was probably of local inflammatory origin, and due to lysosomal and hydrolytic degradation of the membrane. The prolonged local inflammation could reduce the biomechanical properties of the callus and explain that the larger callus area did not result in superior strength. Sedimentation reaction, blood platelets, white blood cell count and differential count revealed no difference among groups, thus denying the possibility of a sustained measurable systemic inflammatory reaction caused by membrane implantation.

The weakness of our study is the low number of experimental animals denying significant conclusions regarding biomechanical properties. Also, serial blood flow measurements and serial serum cytology would have added useful information about the membrane degradation process. The strength of our study in comparison to previous studies is that we use a clinical relevant model and compare the combined action of membrane and rhBMP-2 with membrane alone and rhBMP-2 implantation alone. We thus identified that membrane implantation added no benefit in comparison to rhBMP-2 alone. Further, we added an unbiased bone blood flow estimate to the experimental endpoints.

In summary, interfragmentary deposition of rhBMP-2 delivered in a hyaluronan gel carrier combined with implantation of an absorbable barrier membrane in a clinically relevant model of compromised healing prevented nonunion formation, but was unable to improve biomechanical properties in comparison to rhBMP-2 treatment alone. Implantation of barrier membrane alone could not prevent non-union formation.

\section{Aknowledgements}

Gore \& Associates, Flagstaff, AZ, USA donated the Gore Resolut XT6 ${ }^{\circledR}$ free of charge, and Yamanouchi Europe BV, Leiderdorp, The Netherlands donated rhBMP-2 free of charge. Plates for stabilisation of osteotomies were donated free of charge by Synthes A/S, Hoersholm, Denmark.

No benefits in any form have been received or will be received from a commercial party related directly or indirectly to the subject of this article.

\section{REFERENCES}

[1] G. E. Friedlaender, C. R. Perry, J. D. Cole, S. D. Cook, G. Cierny, G. F. Muschler, et al., "Osteogenic Protein-1 (Bone Morphogenetic Protein-7) in the Treatment of Tibial Nonunions,” The Journal of Bone \& Joint Surgery, Vol. 83-A, Suppl. 1(Pt 2): 2001, pp. S151-S158.

[2] S. Govender, C. Csimma, H. K. Genant, A. ValentinOpran, Y. Amit, R. Arbel, et al., "Recombinant Human Bone Morphogenetic Protein-2 for Treatment of Open Tibial Fractures: A Prospective, Controlled, Randomized Study of Four Hundred and Fifty Patients," The Journal of Bone \& Joint Surgery, Vol. 84-A, No. 12, 2002, pp. 2123-2134.

[3] T. Sibai and E. F. Morgan, T. A. Einhorn, "Anabolic Agents and Bone Quality,” Clinical Orthopaedics and Related Research, Vol. 469, No. 8, 2011, pp. 2215-2224. http://dx.doi.org/10.1007/s11999-010-1722-9

[4] A. Linde, P. Alberius, C. Dahlin, K. Bjurstam and Y. Sundin, "Osteopromotion: A Soft-Tissue Exclusion Principle Using a Membrane for Bone Healing and Bone Neogenesis,” Journal of Periodontology, Vol. 64, Suppl. 
11, 1993, pp. 1116-1128.

http://dx.doi.org/10.1902/jop.1993.64.11s.1116

[5] R. P. Meinig, "Clinical Use of Resorbable Polymeric Membranes in Bone Defects," Orthopedic Clinics of North America, Vol. 41, No. 1, 2010, pp. 39-47. http://dx.doi.org/10.1016/j.ocl.2009.07.012

[6] H. C. Brownlow and A. H. Simpson, "Metabolic Activity of a New Atrophic Nonunion Model in Rabbits," Journal of Orthopaedic Research, Vol. 18, No. 3, 2000, pp. 438442. http://dx.doi.org/10.1002/jor.1100180316

[7] A. A. Reed, C. J. Joyner, H. C. Brownlow and A. H. Simpson, "Human Atrophic Fracture Non-Unions Are Not Avascular," Journal of Orthopaedic Research, Vol. 20, No. 3, 2002, pp. 593-599. http://dx.doi.org/10.1016/S0736-0266(01)00142-5

[8] H. Eckardt, K. S. Christensen, M. Lind, E. S. Hansen, D. W. Hall and I Hvid, "Recombinant Human Bone Morphogenetic Protein 2 Enhances Bone Healing in an Experimental Model of Fractures at Risk of Non-Union,” Injury, Vol. 36, No. 4, 2005, pp. 489-494. http://dx.doi.org/10.1016/j.injury.2004.10.019

[9] M. L. Radomsky, A. Y. Thompson, R. C. Spiro and J. W. Poser, "Potential Role of Fibroblast Growth Factor in Enhancement of Fracture Healing," Clinical Orthopaedics and Related Research, Suppl. 355, 1998, pp. S283S293. http://dx.doi.org/10.1097/00003086-199810001-00029

[10] J. R. S. Hales, "Radioactive Microsphere Techniques for Studies of the Circulation," Clinical and experimental pharmacology \& physiology. Supplement, Vol. 1, 1974, pp. 31-46.

[11] H. E. Stender, S. Z. He, V. E. Hjortdal, D. Kjolseth and K. Soballe, "Distribution of Blood Flow in Normal and Arthritic Joints. Role of Arteriovenous Shunting Studied in Growing Dogs,” American Journal of Physiology-Heart and Circulatory Physiology, Vol.262(1 Part 2), 1992, pp. H38-H46.

[12] P. Ruegsegger, B. Koller and R. Muller, “A Microtomographic System for the Nondestructive Evaluation of Bone Architecture," Calcified Tissue International, Vol. 58, No. 1, 1996, pp. 24-29. http://dx.doi.org/10.1007/BF02509542

[13] M. S. Tonetti, P. Cortellini, J. E. Suvan, P. Adriaens, C. Baldi, D. Dubravec, et al., "Generalizability of the Added Benefits of Guided Tissue Regeneration in the Treatment of Deep Intrabony Defects. Evaluation in a Multi-Center Randomized Controlled Clinical Trial," Journal of Periodontology, Vol. 69, No. 11, 1998, pp. 1183-1192. http://dx.doi.org/10.1902/jop.1998.69.11.1183

[14] R. Dimitriou, G. I. Mataliotakis, G. M. Calori and P. V. Giannoudis, "The Role of Barrier Membranes for Guided Bone Regeneration and Restoration of Large Bone Defects: Current Experimental and Clinical Evidence,” BMC Medicine, Vol. 10, 2012, p. 81. http://dx.doi.org/10.1186/1741-7015-10-81

[15] G. Zellin and A. Linde, "Treatment of Segmental Defects in Long Bones Using Osteopromotive Membranes and Recombinant Human Bone Morphogenetic Protein-2. An Experimental Study in Rabbits," Journal of Plastic Surgery and Hand Surgery, Vol. 31, No. 2, 1997, pp. 97-104. http://dx.doi.org/10.3109/02844319709085475

[16] A. Linde and E. Hedner, "Recombinant Bone Morphogenetic Protein-2 Enhances Bone Healing, Guided by Osteopromotive e-PTFE Membranes: An Experimental Study in Rats," Calcified Tissue International, Vol. 56, No. 6, 1995, pp. 549-553. http://dx.doi.org/10.1007/BF00298588

[17] N. F. Farso, "Guided Bone Induction-A Method in the Treatment of Diaphyseal Long Bone Defects,” 1992. 